\title{
MANNOSE BINDING LECTIN (MBL) LEVELS PREDICT LUNG FUNCTION DECLINE IN SEVERE ASTHMA
}

Ilonka H. van Veen, MD*, Pieter S. Hiemstra, PhD*, Anneke ten Brinke, MD, PhD\#, Anja Roos, PhD", Renate M. Verhoosel*, Jaap K. Sont, PhD+, Peter J. Sterk, MD, PhD*, Klaus F. Rabe, MD, PhD* and Elisabeth H. Bel, MD, PhD*

*Dept of Pulmonology, Leiden University Medical Center, The Netherlands

\#Dept of Pulmonology, Medical Center Leeuwarden, Leeuwarden, The Netherlands

"Dept of Nephrology, Leiden University Medical Center, The Netherlands

${ }^{+}$Dept of Medical Decision Making, Leiden, The Netherlands

WINNING ABSTRACT: There is increasing evidence that activation of the complement system in asthma contributes to ongoing inflammation, tissue damage and airway remodeling. Mannose binding lectin (MBL) is a pattern recognition molecule that serves as the key mediator of the lectin pathway of complement activation. MBL levels are genetically determined and vary widely amongst individuals. In the present study we hypothesized that high MBL levels in asthma are associated with increased loss of lung function over time, as a consequence of inflammatory tissue damage. We measured serum MBL levels by ELISA in 68 patients with severe asthma and prospectively determined the change in post-bronchodilator (pb) FEV1 over a mean period of 5.7 years. The relationship between MBL and change in pbFEV1 ( $\triangle F E V 1)$ was analysed using (multiple) regression analysis and corrected for possible confounders (age, sex, age of onset, asthma duration, and pbFEV1). The median (range) MBL level was $332(10.8-3587) \mathrm{ng} \cdot \mathrm{ml}^{-1}$. MBL was significantly associated with $\Delta \mathrm{FEV} 1(\mathrm{p}<0.04)$. Patients with a high MBL level $\left(\geqslant 332 \mathrm{ng} \cdot \mathrm{ml}^{-1}\right.$ ) had an increased risk of lung function decline compared to those with low MBL levels (OR (CI): 3.16 (1.14-8.79), $\mathrm{p}=0.027)$; the excess decline being $42 \mathrm{ml}^{-y^{-1}}(\mathrm{p}=0.01)$. We conclude that a high MBL level is associated with an increased decline in lung function in patients with severe asthma. MBL might provide a clue towards better understanding of the pathophysiology of ongoing inflammation and subsequent decline in lung function of patients with severe asthma.

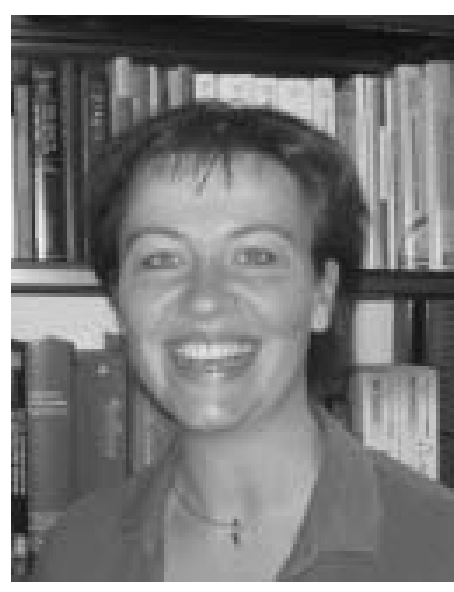

Ilonka H. van Veen

Dept of Pulmonology, Leiden University Medical Center, Leiden, The Netherlands

\section{MY JOB AND THE UNIT IN WHICH I WORK}

I am currently working as a pulmonologist and $\mathrm{PhD}$ fellow in the Dept of Pulmonology at the Leiden University Medical Center (Leiden, the Netherlands). I started my PhD on difficultto-treat asthma in 2002 together with Prof. Elisabeth Bel who is my mentor and has been working in this field for a long time with several other PhD fellows. I also work with Prof. Klaus Rabe, head of our department, Prof. Peter Sterk and Prof. Pieter Hiemstra, who are all involved in asthma research and are collaborating to investigate clinical, physiological and immunological features of this disease.

The Leiden University Medical Center is one of the eight university medical centres in the Netherlands. The hospital is involved in patient care, research, student education and specialist training. The Dept of Pulmonology consists of an outpatient clinic, a clinical ward, a lung function laboratory and a laboratory for respiratory cell biology and immunology. The research performed in our department is mainly focused on the pathogenesis and treatment of asthma and chronic obstructive pulmonary disease (COPD). Novel techniques are also studied in the staging of lung cancer with endosonography of the mediastinum.

\section{MY RESEARCH AS PART OF MY WORKING GROUP/ RESEARCH TEAM AND MY WINNING ABSTRACT AS PART OF MY RESEARCH}

My $\mathrm{PhD}$ focuses on predictors of disease outcome in patients with difficult-to-treat asthma. I am participating in a research project that was initiated in 1998 by Anneke ten Brinke and Elisabeth Bel. The aim of this study performed in our department was to identify different phenotypes of difficultto-treat asthma and to prospectively follow this group of patients to study disease outcome. A total number of 136 patients were recruited from 10 different hospitals in the western part of the Netherlands. All patients underwent an extensive diagnostic protocol, including blood tests, sputum induction, lung function measurements, measurement of

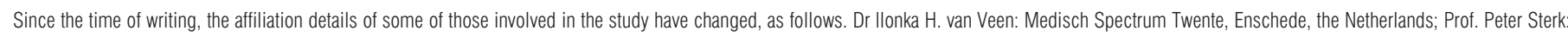
Academic Medical Center, Amsterdam, the Netherlands; Prof. Elisabeth Bel: Academic Medical Center. 
exhaled nitric oxide, computed tomography sinus scanning, $\mathrm{pH}$ measurement of the oesophagus and the completion of several questionnaires. Cross-sectional analysis of these data resulted in the publication of a number of papers describing different phenotypes of difficult-to-treat asthma, such as patients with persistent airflow limitation and frequent exacerbations [1, 2].

One of the main aims of my $\mathrm{PhD}$ is to identify the risk factors of an accelerated decline in lung function. It has been demonstrated that patients with asthma have an accelerated decline in lung function over time [3]. This accelerated decline can result in persistent airflow limitation, which is a common finding in patients with difficult-to-treat asthma ( $\sim 50 \%$ in our cohort) and has been associated with increased morbidity and mortality [4]. However, it is not known which patients are at risk of having an increased loss of lung function over time despite treatment with inhaled or oral corticosteroids. Identification, at an early stage, of those patients at risk of developing persistent airflow limitation is clinically important, since it could positively influence the management of this group of patients, for example, by selecting them to participate in novel drug trials in order to improve asthma outcome. In 2004, we were able to reassess our unique cohort of patients with difficult-to-treat asthma to determine their lung function decline during the previous 6 yrs and to identify possible predictors at an accelerated decline in lung function. The abstract presented at the European Respiratory Society Congress 2006 in Munich (Germany) shows the results of this study.

\section{THE IMPACT OF MY WORK ON CLINICAL OR RESEARCH PRACTICE}

The research in our department on difficult-to-treat asthma will continue to focus on determining different phenotypes of this disease. Patients with difficult-to-treat asthma do not respond well to standard treatment with inhaled or oral corticosteroids and have persistent symptoms, recurrent exacerbations or persistent airflow limitation. These patients need novel treatment strategies or novel anti-asthma drugs to improve their asthma control, but the major challenge is to find the right treatment strategy for the right group of patients. Since difficult-to-treat asthma is a very heterogeneous disease with patients presenting with different clinical, physiological and inflammatory features, it is not to be expected that they will all benefit from the same (novel) treatments to the same extent. Identifying subgroups of patients that have important characteristics in common with regard to disease outcome is necessary to unravel the pathogenesis of their disease and to develop phenotype-specific anti-asthma treatments.

\section{REFERENCES}

1 ten Brinke A, Zwinderman AH, Sterk PJ, Rabe KF, Bel EH. Factors associated with persistent airflow limitation in severe asthma. Am J Respir Crit Care Med 2001; 164: 744-748.

2 ten Brinke A, Sterk PJ, Masclee AA, et al. Risk factors of frequent exacerbations in difficult-to-treat asthma. Eur Respir J 2005; 26: 812-818.

3 Lange P, Parner J, Vestbo J, Schnohr P, Jensen G. A 15-year follow-up study of ventilatory function in adults with asthma. N Engl J Med 1998; 339: 1194-1200.

4 Hansen EF, Phanareth K, Laursen LC, Kok-Jensen A, Dirksen A. Reversible and irreversible airflow obstruction as predictor of overall mortality in asthma and chronic obstructive pulmonary disease. Am J Respir Crit Care Med 1999; 159: 1267-1271. 